\title{
Research on Cross-border Design Language in New Media Time Visual Communication Design
}

\author{
Hui Huang ${ }^{1, a^{*}}$, Junjian Liu ${ }^{2, b}$ \\ ${ }^{1}$ School of Art and Design, Jingdezhen Ceramic Institute, Jingdezhen, 333004, China \\ ${ }^{2}$ Jingdezhen Ceramic Vocational Technical College, Jingdezhen, 333400, China \\ a114249216@qq.com, b48858523@qq.com \\ *Corresponding author
}

Keywords: new media, visual communication design, cross-border design

\begin{abstract}
In the face of changes in new times and new media, the visual communication design is faced with transition and development. In the paper, it analyzes the development motivation (catalysis of digital technique, intersection and integration of design boundary, collaboration of operating type) and summarizes the cross-border design language (combination with wearable technology, combination with streaming media and combination with mobile terminal) of heterogeneous permeation in the visual communication design by taking the generation of cross-border design and the value of visual communication cross-border design as the starting point.
\end{abstract}

\section{Introduction}

With the progress of the times, the visual communication design is faced with transition and development, and it is faced with unprecedented impact as well. Traditional plane media needs to accept challenges and requires designers to face changes of the new times and new media, abandon the inherent thinking model, design method and representation method, actively use new media and new technologies, examine existing visual communication design system again, extract essence adapting the new times, abandon elements impeding the cross-border of the visual communication design to new media and integrate the concept of visual communication design organically again.

\section{Cross-border}

\subsection{Generation of cross-border design}

The application of computer doesn't replace human brain thinking computation; computer typing doesn't replace people's handwriting; and the occurrence of digital media doesn't extinct print media. Just as we said in the above: "new" things and "old" things should not be in opposition to each other; both of them interact to play their characteristics and make them produce more merits. Therefore, the visual communication design taking presswork as the major representation form is not really without value completely under the present information social environment, and it can still deliver information effectively.

In the present art field, the boundary between each subject is very dim and makes the identity of each subject figures be very complex. It is very common that a person is both a designer and an artist, which designs with art skills and technical performance and examine art with the design vision. With continuous new meanings in both respects, the designed work is with both artistic attribute and traditional classical aesthetic taste, and the artistic work is with both pioneer spirit and constructivism. In general, such condition is driven by the application of cross-subject knowledge, while such cross-subject intersection can produce good effects, which is a design mean used by visual communication designers frequently at present. 


\subsection{Value of visual communication cross-border design}

The present visual communication design not only needs the complete coverage of the subject knowledge, but also needs to increase the height and learn other fundamental knowledge of other large subjects. The design should combine with different subjects at different heights to simulate better creative and visual methods, such as, psychology, sociology, bionics, anthropology, sociology, etc. Under comprehensive consideration, cross-border design is more reasonable and complies with individual demands better.

Nowadays, new media develops at a high speed; traditional communication methods update continuously and have higher requirements on designers, which highlights the "new" in the characteristics of the designer industry particularly acute. Cross-border is the requirement of the times and also the direction moving viewers at the most. Poster design in a new morphology is a butt joint of traditional visual communication design and new media. Two dimensions changes to three dimensions even four dimensions, dynamic and instantaneous, opening a new interface and improving the expressive force and visual tension of visual communication design.

\section{Development motivation of visual communication cross-border design}

\subsection{Catalysis of digital technique}

Design is liberated from complex and fussy hand labor with the birth of computer and other aided design, which overturns the original design creative method, makes the design field develop unprecedentedly, makes previous fantastic representation method become reality and improves the integral design level greatly. To design with software not only reduces the drawing time, but also beautifies visual effects, which adds new elements to the design form and upgrades the monotonous two-dimensional representation method to multi-dimensional, just as the idea advocated by the pioneer of modern design education - Bauhaus: “design is a combination of art and technology”. The development of technology inevitably drives the revolution of design. The development of deduction in electronic form, virtual image technology, network communication and other media enrich the communication form of digital information visual language greatly, and these breakthroughs have active development factors for the development of relevant professions, the continuous creation of dimensional development and the cultivation of design talents.

\subsection{Intersection and integration of design boundary}

The blend of various professional fields is displayed in physical environment design, indoor design, exhibition design, industrial design, building design, costume design, public art and virtual multi-media design, animation design, video design and web design; these things have relationship with the most basic visual communication design more or less and display the extension in design vividly and intuitively. In a manner of speaking, the "cross-border" property has permeated in each professional field, which is not only the external three-dimensional space demonstration of design, but also an aggregation art with kinds of professional design ideas within it.

Secondly, the intersection is also displayed on the blend of subjects. Cross-border design has broken through the mode of the previous traditional design science and fine arts; it starts to involve in the subject area of psychology, philosophy and literature, develop analysis and research on principles and theories and make boring theoretical knowledge be displayed through vivid design form. Furthermore, some subjects as means, such as, fine arts, psychology, philosophy and others are mature subjects with scientific system concept and special method, which provide new regulated research form for the building and research of cross-border design and promote the exploration between subjects; additionally, on the basis of the development, the intersection strengthen the appeal of subjects intersection idea and design development, forming creative advantages in cross-border design.

\subsection{Collaboration of operating type}

The collaboration of operating type means a collaborative work and research method between 
professions in the design field. Although the professional contents and natures of each subject are different, they have potential dependence relation between each other, for example, the visual communication design and product design is indispensable; the external packing of products needs the support of graphic elements and written messages of the visual communication profession, while the packing design itself depends on the product internal formation; both are indispensable and an expression of collaboration. Professions contact each other more or less during the process from program planning, modification to implementation; they trigger communications and integration in design without noticing. This is the so-called collaboration of operating type.

\section{Cross-border Design Language of Heterogeneous Permeation in the Visual Communication design}

Various new technologies are kept coming out with the rapid development of digital product and electronics technology. They can bring people brand-new experience through proper carriers, and visual communication design is just their platform. Visual communication cross-border design will combine the characteristics of new technologies more closely, fully display new media characteristics and design works with era significance.

\subsection{Combination with wearable technology}

Wearable technology is a new technology developed in recent years and a joint innovation between high-tech and design. Apart from completing products' traditional functions, it provides more special functions for people who wear it. For example, GOOGLE glasses completes giving directions, photographing, shooting and other functions with the built-in GPS, sensor and camera, etc.; SEAL swimming monitor neck ring can trace the swimmer's safety condition at any time, send data to the guardian and can reduce swimming accidents of children and the aged effectively. For wearable equipment, how to deliver rich information in such a small screen interface is more urgent than new interface and new expressional way. (E.g.Figure1)

\subsection{Combination with streaming media}

Streaming media is a technological mean realizing network real-time video transmission after compressing a series of media data through network segmented transmission for enjoyment. The generation of streaming media has solved the question of network transmission speed completely and added voice and dynamic images for rigid static web pages; compared with traditional pictures, they are more accurate with richer information. For visual communication design, the cross-border combination with streaming media is a brand-new try. Streaming media will bring about more extension and possibility and enrich people’s sense of sight continuously.

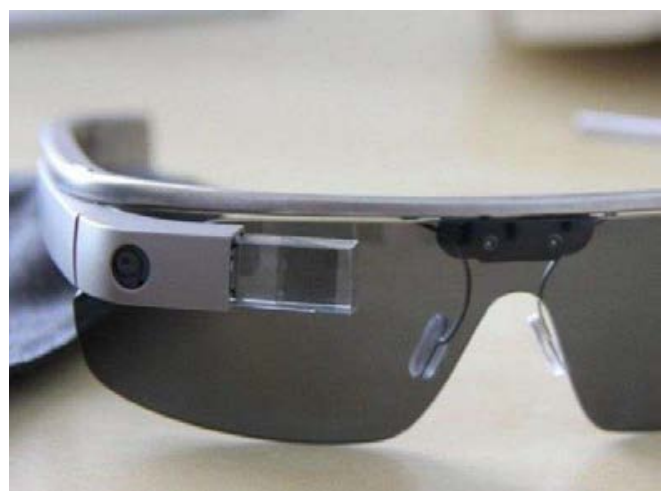

Figure 1 GOOGLE glasses with the built-in GPS 


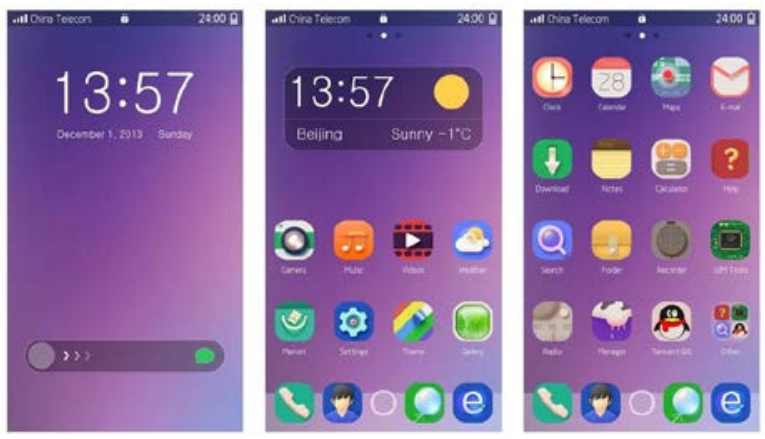

Figure 2 mobile interface

\subsection{Combination with mobile terminal}

Our mobile phone could only call ten years ago. Nowadays, mobile terminals taking mobile phone have occupied our life powerfully; navigation, shopping, making friends, news even works can be completed on the mobile terminals accompanying with various software on terminals. The experience and feelings given by software interface application to customers is needed to complete by visual communication design. The design must cooperate with the software functions, audiences, aesthetic nature and interaction; thus, users' usage experience can be improved effectively, and then, the product usage rate and click rate are increased. Thus it can be seen that visual communication design has not been dwelled on the traditional visual space; the new generation of digital technology helps the design break through the traditional design mode in delivery way, delivery method, delivery effect, usage experience and other respects and brings visual communication design to a brand-new filed. The cross-border combination of design and new technologies becomes the new direction of visual communication development. (E.g. Figure2)

\section{Summary}

Visual communication cross-border design is related to the present big bang theory and knowledge quantity explosion. There are more intersections between subjects. The classification of subjects merges gradually; instead of developing respectively, they turn to mutual learning and crossing development. It is the development trend of science and technology, so as visual communication design. If wanting to develop, it must learn from each other with other subjects, draw on each other's strength and develop from a single subject to diversification and collaboration. It is not only an indisputable fact in nowadays society, but also the trend.

\section{References}

[1] ZhangYang, Crossover and integration_-new reflections on visual communication design in the context of the digital information era, Design (2015.8) P82-83

[2] LiuYu long, Cross - border Convergence —_The Influence of Pan - media Times on Graphic Design ,Journal of Nanjing Arts Institute(Fine Arts \& Design) (2012.2)P161-162

[3] PengMei, Dimensional expansion and application research in graphic design, Beijing University of technology masteral dissertation （2015） P33-36

[4] MaJing, The Competition of Space - Research on the Plane Art in Public Space, China Academy of Fine Arts masteral dissertation （2010） P10-12

[5] LiXiang, On the Expression of Graphic Art in Public Space, Art Education Research (2011)P69 